\title{
Nanoscience and Aptamer Technology for Point of Care Diagnostics
}

\author{
Tarun Kumar Sharma ${ }^{1,2 *}$ and Ravi Shukla ${ }^{2,3 *}$ \\ ${ }^{1}$ Center of Biodesign \& Diagnostics, Translational Health Science and Technology Institute, Haryana, India \\ ${ }^{2}$ Centre for Advanced Materials \& Industrial Chemistry, RMIT University, Australia \\ ${ }^{3}$ Health Innovation Research Institute, RMIT University, Australia
}

Received: March 12, 2014; Accepted: March 13, 2014; Published: March 14, 2014

*Corresponding author: Ravi Shukla, Centre for Advanced Materials \& Industrial Chemistry, and Health Innovation Research Institute, RMIT University, Melbourne 3001 VIC, Australia; Tel: 0061-3-9925-2970; Fax: 0061-3-9925-2882; E-mail: ravi.shukla@rmit.edu.au

Tarun Kumar Sharma, Center of Biodesign \& Diagnostics, Translational Health Science and Technology Institute, Gurgaon, Haryana-122016, India, Email:tarun@thsti.res.in

Food and water are considered as vital commodities as it is equally important for everyone. Contamination of food and water by variety of microbial pathogens (viruses, bacteria and protozoan etc.), toxins, heavy metals, organic pollutants (pesticides, dyes, and other chemical carcinogens) and adulterants is a matter of serious concern. There are a number of diagnostics tools in place which offer detection of aforementioned pathogens and contaminants with variable degree of sensitivity and specificity including but not limited to molecular assays, biophysical techniques and analytical methods [1-3]. Unfortunately, these methods are limited in applicability for onsite detection as most of these methods require sophisticated instrumentation and trained personal. In other words, these methods fail to meet the "ASSURE" criteria suggested for an ideal point-of-care diagnostic test that demands affordability, sensitivity and specificity; is rapid and robust; and does not require sophisticated equipment [4].

Emergence of nanotechnology based detection is currently considered as rising hope for designing smart sensors, which not only have the potential to recognize biological and chemical threat in real time but can also perform their detection with extremely high sensitivity. Due to the excellent plasmon absorption of noble metal nanoparticles (NPs), especially of gold and silver, a variety of optical sensors based on functional noble metal NPs have been developed. The sensors are designed by choosing an appropriate ligand to be functionalized on the metal NPs. Upon specific interactions of the ligand with the target analyte; the system undergoes a visible color change which can also be read spectroscopically for quantitative estimation. Among nanoparticles (NPs), gold nanoparticles (GNPs) and quantum dots (QDs) are commonly used as optical sensors, because of their color and fluorescence properties. GNPs present different colors, from red to purple/ blue depending on the interparticle distance in particular reaction. Other optical sensors are based on photoluminescence changes of QDs or dyes [5]. Although this nanoscience tool offers remarkable sensitivity for a pointof-care diagnostics with the aim to meet "ASSURE criteria"; without incorporation of an appropriate molecular recognition element such as antibody or aptamer which binds its target with high affinity and selectivity, the application of nanomaterials for optical sensors seems to be a futile exercise.

Aptamers are synthetic ssDNA, RNA or peptide which can be evolved through a process of in vitro evolution known as SELEX (Systemic Evolution of Ligands by Exponential Enrichment). Aptamers are emerged as chemical rival of antibodies which binds to its target with high affinity and specificity and have several advantages over antibody such as no need of animal for generation, ease in functionalization with nanomaterials, flourophore and catalytic ribozymes or quadruplex, less or almost negligible batch to batch variation. Taken together, aptamers are considered as potential molecular recognition elements (MREs), a typical part of biosensor which binds to analyte and gives a visible signal with the help of transducer (signaling element) [6]. When these MREs are coupled to signaling element such as gold nanoparticles or Quatum dots they give visual target specific readout (Red to blue color change in case of GNPs and turn on/turn off fluorescence response in case of QDs). Moreover if the concentration of target analyte in sample is lower than the limit of detection of MREs then the combination of magnetic nanoparticles and capture aptamers can concentrate the amount of analyte in a sample tube which facilitate the detection by other set of aptamers in sandwich format [7]. Further application of aptamers with GNPs can be used for dot blot assay and sensitivity of Pico molar range could be achieved when detection limit were enhanced with silver. This field of aptamer technology along with nanoscience is swiftly evolving to overcome the limitation of nanoscience. Moreover, when these technologies combined with Surface Enhanced Raman Spectroscopy (SERS), they can evince their potential in single cell/single molecule detection. Though these technologies have enormous potential but still they are in infant stage. If their potential has explored to a limit they can open a new vista in the field of point of care nanosensing and can also help in environmental monitoring, surveillance and decision making. Therefore, it is clear that in order to deliver an efficient 
point of care diagnostics, an amalgamation of nanoscience with emerging diagnostic platforms that include but not limited to aptamer technology, SERS, catalytic G quadruplex, ribozymes, protein scaffoldings or phage display screening technology [2-8] would be necessary.

\section{References}

1. Tan D, He Y, Xing X, Zhao Y, Tang H, et al. (2013) Aptamer functionalized gold nanoparticles based fluorescent probe for the detection of mercury (II) ion in aqueous solution. Talanta 15(113): 26-30.

2. Olivier L, Campo F, Xavier Munoz F (2006) Pathogen detection: A perspective of traditional methods and biosensors. Biosenosors and Bioelectronics, 22(2007): 1205-1217.

3. Wu WH, Yue ML, Wang Y, Ouyang HX, Wang L, et al. (2012) Aptasensors for rapid detection of Escherichia coli 0157:H7 and Salmonella typhimurium. Nanoscale Res Lett 7(1): 658.

4. Pai NP, Vadnais C, Denkinger C, Engel E, Pai M (2012) Point-of-care testing for infectious diseases: diversity, complexity, and barriers in low- and middle-income countries. PLOS Med 9(9): e1001306.

5. Riu J, Maroto A, Rius FR (2006) Nanosensors in enviornmental analysis. Talanta 69(2): 288-301.

6. Sharma TK, Shukla R (2014) Nucleic Acid Aptamers as an Emerging Diagnostic Tool for Animal Pathogens. Advances in Animal and Veterinary Sciences 2(1): 50-55

7. Bruno JG, Phillips T, Carrillo MP, Crowell R (2009) Plastic-adherent DNA aptamer-magnetic bead and quantum dot sandwich assay for Campylobacter detection. J Fluoresc 19(3): 427-435.

8. Afrasiabi Z, Shukla R, Chanda N, Bhaskaran S, Upendran A, et al. (2010) Nanoscale sensor design via in situ labeling of gold nanoparticles onto protein scaffolds. J Nanosci Nanotechnol 10(2): 719-725. 